\title{
Comparing energy loss phenomenology
}

\author{
M. van Leeuwen*t \\ Utrecht University, PO Box 80000, 3508 TA Utrecht, Netherlands \\ E-mail: m.vanleeuwen1@uu.nl
}

High- $p_{T}$ particle production is suppressed in heavy ion collisions due to parton energy loss in dense QCD matter. Here we present a systematic comparison of two different theoretical approximations to parton energy loss calculations: the opacity expansion and the multiple-soft scattering approximation for the simple case of a quark traversing a homogeneous piece of matter with fixed length (the TECHQM 'brick problem'), with focus on the range of parameters that is relevant for interpreting RHIC measurements of high- $p_{T}$ hadron suppression.

High-pT Physics at LHC -09

February 4 - 72009

Prague, Czech Republic

\footnotetext{
* Speaker.

$\dagger$ The author is indebted to the TECHQM collaboration for many useful discussions about the different energy loss schemes.
} 
One of the important findings at RHIC is that high transverse momentum $\left(p_{T}\right)$ hadron production in central $\mathrm{Au}+\mathrm{Au}$ collisions is suppressed compared to (properly scaled) $\mathrm{p}+\mathrm{p}$ collisions $[1,2]$. This suppression can be attributed to energy loss of high- $p_{T}$ partons that traverse the hot and dense medium formed in these collisions. An important goal of the study of heavy ion collisions is therefore to determine the medium density from the measured suppression by comparing the measurements to sufficiently detailed calculations of parton energy loss and hadronisation in the medium.

Four different theoretical descriptions of radiative energy loss in hot and dense QCD matter are generally used. Two of the descriptions: the opacity expansion and multiple soft scattering approach, have been shown to be different approximations of the same basic path-length formalism which was formulated by Baier, Dokshitzer, Muller, Peigne and Schiff (BDMPS) [3] and independently by Zakharov [4]. The higher-twist formalism was formulated by Wang and Guo [5] and starts from higher-twist terms which are closely related to higher twist in Deeply Inelastic Scattering calculations. It is also possible to calculate energy loss in Hard Thermal Loop field theory, as explored by Arnold, Moore and Yaffe (AMY) [6].

For a realistic calculation of final state hadron suppression, the theoretical description of the fundamental energy loss process needs to be supplemented with parton production and fragmentation and a realistic collision geometry. Existing calculations have used various simplified geometries, including fixed (mean) path length, (uniform) hard spheres and Woods-Saxon overlap geometries. Some recent work has been done with realistic hydrodynamic media [7-9].

For a systematic evaluation of the medium density and the uncertainties on the density, we would like to compare the various theoretical frameworks using the same geometry and then vary the assumptions about the geometry in an independent way. Unfortunately, the existing literature does not provide such a systematic comparison.

For example, a long-standing issue is the comparison of the opacity expansion and the multiplesoft gluon emission approximation. The basic energy loss kernels for both formalisms have been compared in some detail and it was found that both formalisms could be made to roughly agree [10], but no connection was made to the medium density or temperature. More complete calculations of hadron suppression using the two formalisms have been performed by two independent groups, which makes it difficult to compare the results. For the multiple-soft scattering approach, the PQM model implements a Woods-Saxon geometry where the medium density is assumed to scale with the local binary collision density [11]. It was found that the average transport coefficient $\hat{q}$ that a parton sees is $13.2 \mathrm{GeV}^{2} / \mathrm{fm}$ [12], corresponding to an estimated average medium temperature (see section 1.1 below) of $1 \mathrm{GeV}$. A similar calculation for the opacity expansion (GLV) was performed by Wicks, Horowitz, Djordjevic and Gyulassy [13], assuming the medium density to be proportional to the local participant density. In this calculation, it is found that a medium with about 1400 soft gluons in the transverse plane at mid-rapidity is needed to reproduce the measured nuclear modification factor $R_{A A}[12]$. This value corresponds to a temperature of approximately $370 \mathrm{MeV}$. From this, it seems that medium densities extracted using the two different formalisms are vastly different (a factor 2 in $T$ corresponds to a factor 8 in density). In the following, we will explore some of the possible origins of these differences in detail. 


\section{The TECHQM brick problem}

A general calculation of energy loss effects on high- $p_{T}$ spectra in heavy ion collisions involves not only the energy loss kernel which is calculated in (perturbative) QCD, but also the input parton spectrum, parton fragmentation, and the geometry of the reaction zone. As a result, it can be difficult to judge whether differences in e.g. the obtained medium density/effective temperature are generated by the energy loss formalism itself or some of the additional assumptions.

The 'Theory Experiment Collaboration on Hot Quark Matter' (TECHQM) has proposed a set of benchmark problems to compare different energy loss formalisms, also known as the 'brick problem' [14]. The TECHQM Brick Problem asks to calculate quark energy loss for a fixed-length homogeneous medium (the brick). Calculations are performed for two typical path lengths: 2 and $5 \mathrm{fm}$ and 2 partons energies 10 and $100 \mathrm{GeV}$. The following discussion will be largely based on this problem.

\subsection{Defining a common scale: $T$}

In the following, we will focus on comparing the first order opacity expansion (GLV, singlehard scattering) [15] approach and the multiple-soft (ASW) [10] approach. Energy loss in any scheme is expected to be governed by the medium density $\rho$ and the path length $L$. In the two schemes considered here, three variables are generally discussed: the average momentum kick per radiated gluon $\left\langle k_{T}\right\rangle$, the mean free path $\lambda$ and the path length $L$. For the multiple soft scattering approach, the transport coefficient $\hat{q}=\left\langle k_{T}^{2}\right\rangle / \lambda$ is also often used.

In the following, the temperature $T$ will be used as a common scale. The density of gluons in a QGP (or pure gluon gas) is

$$
\rho_{g}=\frac{16 \zeta(3)}{\pi^{2}} T^{3}=\frac{16 \cdot 1.202}{\pi^{2}} T^{3}=1.94 T^{3} .
$$

For simplicity, we approximate the medium as a pure gluon gas. The gluon-gluon transport cross section $\sigma_{g g}$ (to logarithmic accuracy) is [16]

$$
\sigma_{g g}=\frac{9 \pi \alpha_{s}^{2}}{2 m_{D}^{2}},
$$

where $\alpha_{s}$ is the strong coupling constant and $m_{D}$ is the Debye screening mass $\left(m_{D}=\sqrt{4 \pi \alpha_{s}} T\right.$ for a pure gluon gas). The mean free path is

$$
\lambda_{g}=1 / \sigma_{g g} \rho_{g}=\frac{\pi^{2}}{18 \zeta(3) \alpha_{s}} \frac{1}{T}
$$

We further take $\left\langle k_{T}^{2}\right\rangle=m_{D}^{2}$. This ties all the parameters in the calculations to $T$.

\section{Comparing energy loss - importance of distribution width}

Figure 1, left panel, compares the probability (density) distribution for energy loss $P(\Delta E)$ for the WHDG opacity expansion (black curve) [13] and ASW multiple soft scattering approach (red curve) [10] for a $10 \mathrm{GeV}$ quark and $L=2 \mathrm{fm}$. For this comparison, the medium densities 

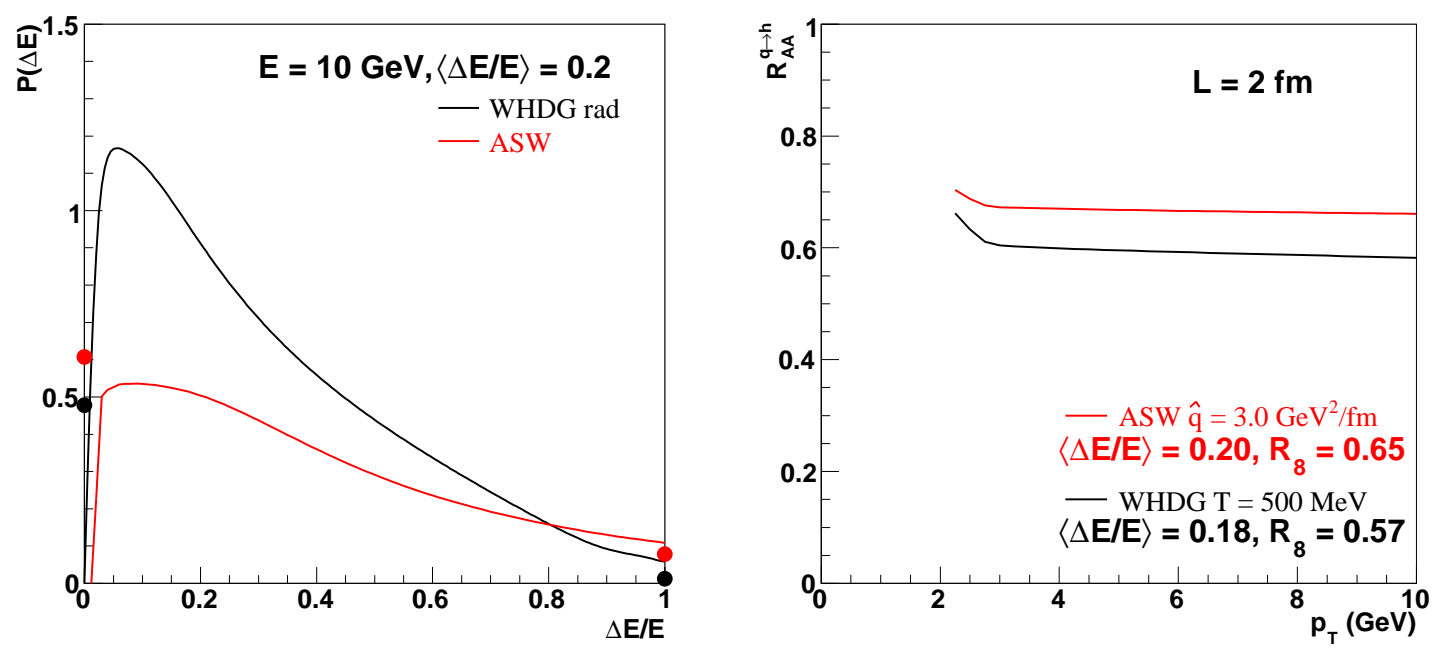

Figure 1: Left panel: energy loss distribution for a quark with $E=10 \mathrm{GeV}$ in a homogeneous medium of $L=2 \mathrm{fm}$ and $\langle\Delta E / E\rangle \simeq 0.2$, from the multiple-soft scattering ASW approach and the WHDG opacity expansion. The points on the left and right axis give the discrete probabilities to lose no energy and to lose the entire energy. Right panel: Nuclear modification factor for pions from quark fragmentation, using the energy loss distributions in the left panel and KKP fragmentation.

have been set to obtain an average fractional energy loss of 0.2 for both models $(T=500 \mathrm{MeV}$ and $\hat{q}=3 \mathrm{GeV}^{2} / \mathrm{fm}$ ). The two different approaches give significantly different energy loss distributions, starting from the probability to lose no energy at all $P(0)$, which is 0.5 for the opacity expansion and 0.6 for the multiple soft scattering approximation. The continuous part the distribution shows correspondingly larger probabilities for WHDG than ASW. In addition, the opacity expansion curve is more strongly peaked towards small energy losses.

A mean fractional energy loss of 0.2 was chosen for Fig. 1, because for a power law spectrum with $n=8$, this is expected to generate $R_{A A} \sim(1-0.2)^{n-1}=0.21$ close to the measured value of $0.2-0.25$ at RHIC $[1,2,12]$. The right panel of Fig. 1 shows $R_{A A}$ calculated for quark fragments, using a realistic (LO pQCD) parton spectrum and KKP fragmentation [17]. The observed values of $R_{A A}^{q}$ is much larger than the naively expected value of 0.21 , due to the broad distribution $P(\Delta E)$. In addition, it is seen that $R_{A A}$ is smaller for the opacity expansion formalism than in the multiplesoft scattering approximation, as one would expect from the smaller value of $P(0)\left(R_{A A}\right.$ has to be greater than or equal to $P(0)$ ).

A better estimate of $R_{A A}$ is the weighted average:

$$
R_{n}=\int_{0}^{1} d \varepsilon(1-\varepsilon)^{n} P(\varepsilon)
$$

where $\varepsilon=\Delta E / E$. The calculated values of $R_{8}$ are shown in the figure and are in approximate agreement with $R_{A A}^{q}$.

\section{Single-hard and multiple-soft scattering}

Figure 2 shows a comparison of energy loss in the opacity expansion and the multiple soft 

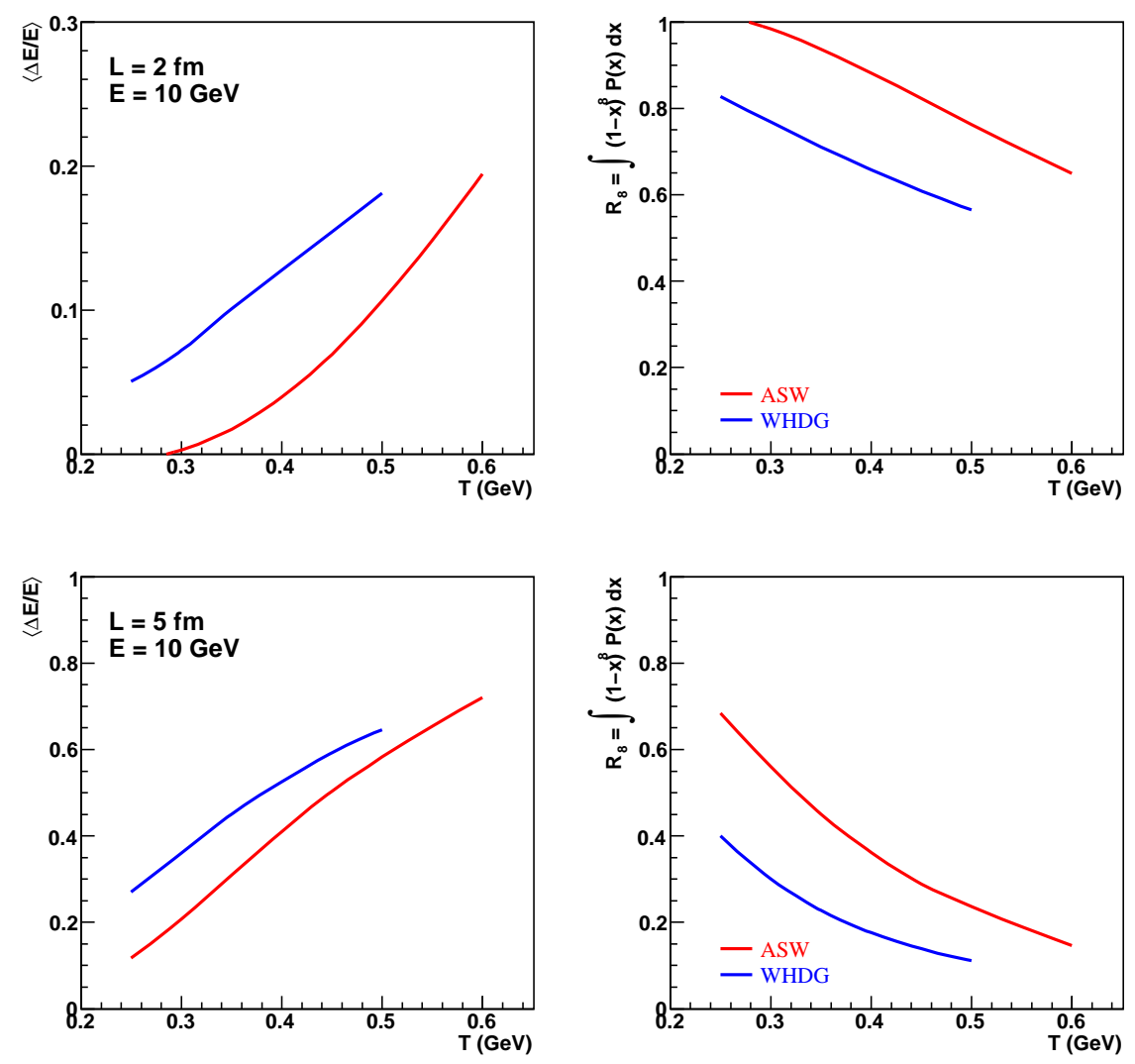

Figure 2: Mean fractional energy loss (left panels) and $R_{8}$ (right panel) as a function of medium temperature for a quark passing through a homogeneous medium of length $L=2 \mathrm{fm}$ (upper panels) and $L=5$ fm (lower panels). The red curves shows the result in the multiple-soft scattering approach (ASW)[10] and the blue curves are for the opacity expansion (WHDG)[13].

scattering formalism as a function of the temperature of the medium. The upper panels show results for $L=2 \mathrm{fm}$ and the lower panels for $L=5 \mathrm{fm}$. The values of $\hat{q}$ for the ASW calculation and $\mu, \lambda$ for the WHDG calculation were calculated using the equations in Section 1.1. It can be clearly seen in the figure that the WHDG formalism typically leads to a larger mean energy loss or smaller $R_{A A}$ for a given temperature. This might be due to the larger impact of the high-energy tail in the energy spectrum of radiated gluons in the opacity expansion than in the multiple soft scattering approximation [10]. The differences are more pronounced at low temperatures and short path lengths than at high temperatures and long path lengths. For $L=2 \mathrm{fm}$, the fractional energy loss reaches 0.2 at $T=500 \mathrm{MeV}$ and $T=600 \mathrm{MeV}$ for WHDG and ASW respectively. The corresponding values of $R_{8}$ are close to 0.6. With a medium length $L=5$, the energy loss is much larger, reaching $R_{8} \sim 0.2$ at $T=375 \mathrm{MeV}$ for WHDG and $T=530 \mathrm{MeV}\left(\hat{q}=1.9 \mathrm{GeV}^{2} / \mathrm{fm}\right.$ ) for ASW. Clearly, large path lengths are needed to reach the value $R_{A A} \sim 0.2$ that is observed at RHIC. The corresponding mean energy loss is also large: $\varepsilon \sim 0.5$.

It is interesting to note that using the brick geometry, the difference between the temperature needed to reach $R_{A A}=0.2$ in the GLV and BDMPS formalism is about a factor 1.5, while some 

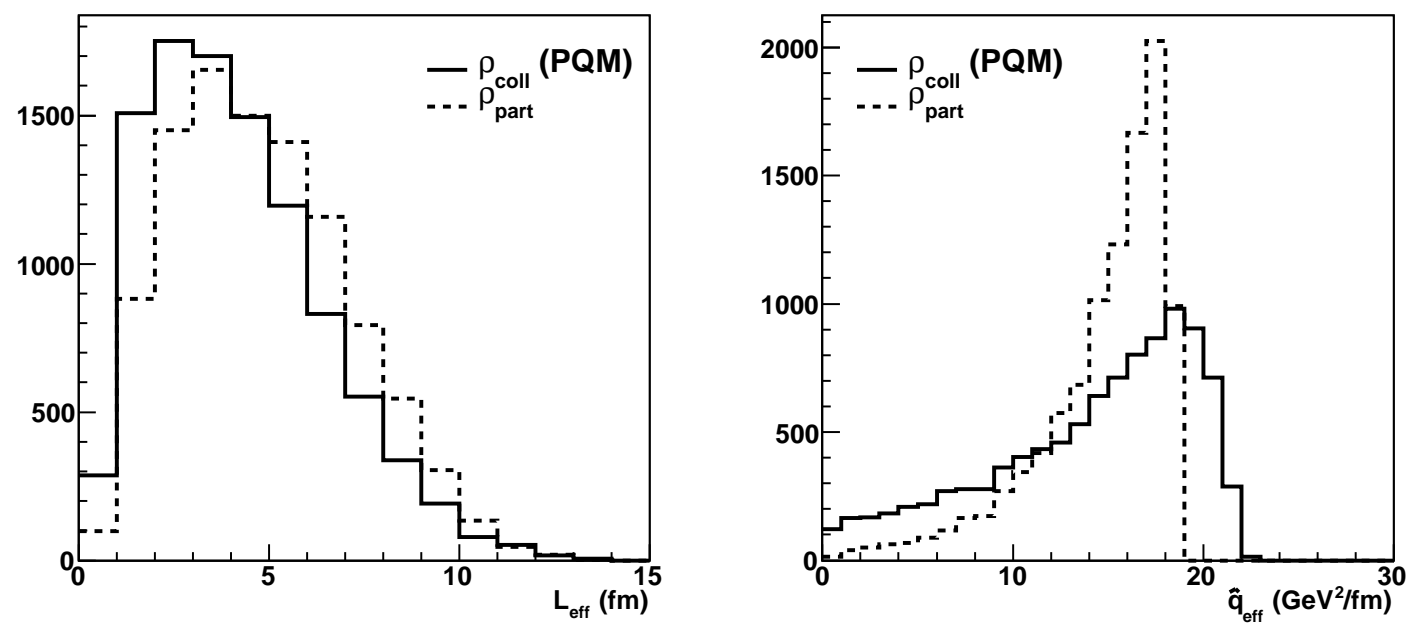

Figure 3: Distributions of effective path length $L_{e f f}$ and transport coefficient $\hat{q}_{e f f}$ in the case where the local medium density is proportional to the density of hard scatterings $\rho_{\text {coll }}$ (solid line) and the density of participant nucleons $\rho_{\text {part }}$ (dashed line) for $0-10 \%$ central $\mathrm{Au}+\mathrm{Au}$ collisions. The proportionality constants for the medium density scaling are given in the text.

earlier studies indicated factors of 2-2.5 (see introduction).

\section{Note on geometry}

The space-time geometry of the collision is an important ingredient of realistic calculations of hadron suppression due to parton energy loss. In the above, a fixed path length and homogeneous medium were used to set up a simple benchmark comparison. Most existing calculations in the literature use somewhat more realistic, parametrised geometries which start from hard spheres or the Woods-Saxon density profile of cold nuclei. Only very recent calculations use realistic geometries from hydrodynamic calculations [7-9].

In particular, the PQM model [11] uses quenching weights from the multiple-soft scattering approximation with a density profile that is proportional to the local density of hard scatterings $\rho_{\text {coll }}$, while the WHDG calculations [13] use the opacity expansion with a density profile that is proportional to the local density of participants $\rho_{\text {part }}$. Figure 3 compares the distributions of effective path lengths $L_{e f f}$ and transport coefficient $\hat{q}$, for a medium medium density scaling with $\rho_{\text {coll }}$ and $\rho_{\text {part }}$. L Leff and $\hat{q}_{\text {eff }}$ are calculated using the same averaging procedure as in the PQM model:

$$
L_{e f f}=\frac{2 I_{1}}{I_{0}}, \hat{q}_{e f f}=\frac{I_{0}^{2}}{2 I_{1}}
$$

with

$$
I_{n}=\int_{0}^{\infty} d u u^{n} \hat{q}(u),
$$

where $\hat{q}(u)$ is the local density and the integral runs along the path of the parton through the medium. The points of origin for the partons follow a $\rho_{\text {coll }}$ distribution in both cases. 
The density distributions as a function of the position the transverse plane $\vec{s}$ and the impact parameter $\vec{b}$ are calculated from the thickness functions $T_{A}$ using

$$
\begin{gathered}
\rho_{\text {coll }}(\vec{s} ; \vec{b})=T_{A}\left(\vec{s}-\frac{1}{2} \vec{b}\right) T_{B}\left(\vec{s}+\frac{1}{2} \vec{b}\right) \\
\rho_{\text {part }}(\vec{s} ; \vec{b})=T_{A}\left(\vec{s}-\frac{1}{2} \vec{b}\right)\left(1-e^{-\sigma T_{B}\left(\vec{s}+\frac{1}{2} \vec{b}\right)}\right)+T_{B}\left(\vec{s}+\frac{1}{2} \vec{b}\right)\left(1-e^{-\sigma T_{A}\left(\vec{s}-\frac{1}{2} \vec{b}\right)}\right) .
\end{gathered}
$$

The terms in brackets in the equation for $\rho_{\text {part }}$ are needed to cut off the naive $\rho_{\text {part }} \propto T_{A}+T_{B}$ in cases where the density in one of the nuclei is close to zero. These terms are the Poisson probability to have at least one struck nucleon in the 'other' nucleus ( $\sigma$ is the total inelastic cross section for a proton-proton collision, $42 \mathrm{mb}$ at RHIC energies).

There is one free scaling parameter in both distributions. For the case of $\rho_{\text {coll }}$ scaling, the parameter has been set to the same value as in the PQM model $\left(k_{\text {coll }}=5 \cdot 10^{6} \mathrm{fm} / A^{2}[11]\right)$. The value for $\rho_{\text {part }}$ was chosen such that the average $\hat{q}_{\text {eff }}$ was similar to the $\rho_{\text {coll }}$ case $\left(k_{\text {part }}=2.3\right.$. $\left.10^{4} \mathrm{fm}^{-1} / A\right)$.

The qualitative difference between the $\rho_{\text {part }}$ geometry and the $\rho_{\text {coll }}$ geometry is that the participant density is more uniform than the collision density. This leads to a larger $L_{\text {eff }}$ and a more sharply peaked distribution for $\hat{q}_{e f f}$ than in the case where the density scales with $\rho_{\text {coll }}$. The corresponding suppression is therefore expected to be larger for a participant density geometry than for the collision density scaling. This may partially explain the differences between the medium density as determined from the WHDG model and from the PQM model.

\section{Discussion and outlook}

A full calculation of hadron suppression due to parton energy loss in heavy ion collisons contains a number of ingredients. Existing calculations have found a large range of different values for the medium density that is needed to describe the measured suppression at RHIC.

When comparing radiative energy loss for a fixed pathlength in a homogeneous medium in the first order approximation of the opacity expansion and the multiple soft scattering approximation, the differences are found to be sizable, with a significant dependence on path length and medium density or temperature. For example, $R_{A A} \sim 0.2$ is reached for a quark in a gluon gas of temperature $T=375 \mathrm{MeV}$ in the opacity expansion and $T=530 \mathrm{MeV}$ for the multiple soft scattering approach. In both calculations, the average energy loss is large (approx. 50\%) and the probability distribution is very broad. This is an important to keep in mind for phenomenolgy, because it means that one cannot think in terms of 'typical' or 'average' energy loss. In addition, the formalisms to calculate energy loss use approximations that are applicable for small fractional energy loss. It would be interesting to estimate the uncertainties from these assumptions.

Another important aspect of energy loss models is the medium density profile. Two different assumptions are commonly found in the literature: the local medium density is either proportional to the local participant density $\rho_{\text {part }}$ or to the local collision density $\rho_{\text {coll }}$. The latter approach leads to shorter effective path lengths and larger fraction of partons that experience a small effective medium density. Both effects lead to a smaller energy loss for a $\rho_{\text {coll }}$ density profile than for a $\rho_{\text {part }}$ profile with the same central density. 
Future work will concentrate on using the different energy loss formalisms in a more realistic geometry. We also aim to extend the comparison to the Higher Twist and Hard Thermal Loop formalisms.

\section{References}

[1] K. Adcox et al. (PHENIX), Suppression of hadrons with large transverse momentum in central $A u+A u$ collisions at $\sqrt{s_{N N}}=130 \mathrm{GeV}$, Phys. Rev. Lett. 88, 022301 (2002), [arXiv:nucl-ex/0109003]

[2] C. Adler et al. (STAR), Centrality dependence of high $p_{T}$ hadron suppression in $A u+A u$ collisions at $\sqrt{s_{N N}}=130 \mathrm{GeV}$, Phys. Rev. Lett. 89, 202301 (2002), [arXiv:nucl-ex/0206011]

[3] R. Baier, Y.L. Dokshitzer, A.H. Mueller, S. Peigne, D. Schiff, Radiative energy loss of high energy quarks and gluons in a finite-volume quark-gluon plasma, Nucl. Phys. B483, 291 (1997), [arXiv:hep-ph/9607355]

[4] B.G. Zakharov, Fully quantum treatment of the Landau-Pomeranchuk-Migdal effect in QED and $Q C D$, JETP Lett. 63, 952 (1996), [ arXiv:hep-ph/9607440]

[5] X.N. Wang, X.f. Guo, Multiple parton scattering in nuclei: Parton energy loss, Nucl. Phys. A696, 788 (2001), [arXiv:hep-ph/0102230]

[6] P. Arnold, G.D. Moore, L.G. Yaffe, Transport coefficients in high temperature gauge theories. I: Leading-log results, JHEP 11, 001 (2000)， [arXiv:hep-ph/0010177]

[7] T. Renk, K. Eskola, Prospects of medium tomography using back-to-back hadron correlations, Phys. Rev. C75, 054910 (2007), [arXiv:hep-ph/0610059]

[8] T. Renk, J. Ruppert, C. Nonaka, S.A. Bass, Jet-quenching in a 3D hydrodynamic medium, Phys. Rev. C75, 031902 (2007), [arXiv:nucl-th/0611027]

[9] G.Y. Qin et al., Radiative jet energy loss in a three-dimensional hydrodynamical medium and high $p_{T}$ azimuthal asymmetry of $\pi^{0}$ suppression at mid and forward rapidity in $A u+A u$ collisions at $\sqrt{s_{N N}}=200 \mathrm{GeV}$, Phys. Rev. C76, 064907 (2007), [arXiv:0705.2575]

[10] C.A. Salgado, U.A. Wiedemann, Calculating quenching weights, Phys. Rev. D68, 014008 (2003), [arXiv:hep-ph/0302184]

[11] A. Dainese, C. Loizides, G. Paic, Leading-particle suppression in high energy nucleus nucleus collisions, Eur. Phys. J. C38, 461 (2005), [arXiv:hep-ph/0406201]

[12] A. Adare et al. (PHENIX), Quantitative Constraints on the Opacity of Hot Partonic Matter from Semi-Inclusive Single High Transverse Momentum Pion Suppression in Au+Au collisions at $\sqrt{s_{N N}}=200 \mathrm{GeV}$, Phys. Rev. C77, 064907 (2008), [arXiv:0801.1665]

[13] S. Wicks, W. Horowitz, M. Djordjevic, M. Gyulassy, Elastic, Inelastic, and Path Length Fluctuations in Jet Tomography, Nucl. Phys. A784, 426 (2007) , [arXiv:nucl-th/0512076]

[14] TECHQM Collaboration, https://wiki.bnl.gov/techqm/index.php/partonic_energy_loss

[15] M. Gyulassy, P. Levai, I. Vitev, Jet quenching in thin quark-gluon plasmas. I: Formalism, Nucl. Phys. B571, 197 (2000), [arXiv:hep-ph/9907461]

[16] R. Baier, D. Schiff, Deciphering the properties of the medium produced in heavy ion collisions at RHIC by a $P Q C D$ analysis of quenched large $p_{T} \pi^{0}$ spectra, JHEP 09, 059 (2006), [arXiv:hep-ph/0605183]

[17] B.A. Kniehl, G. Kramer, B. Potter, Testing the universality of fragmentation functions, Nucl. Phys. B597, 337 (2001), [arXiv:hep-ph/0011155] 